OPEN ACCESS

Edited by:

Guiyin Xu,

Massachusetts Institute of

Technology, United States

Reviewed by:

Tianyu Liu,

Virginia Tech, United States

Wei Luo,

Tongji University, China

*Correspondence:

Xiaogang Zhang

azhangxg@nuaa.edu.cn

Specialty section:

This article was submitted to Electrochemical Energy Conversion and Storage,

a section of the journal Frontiers in Energy Research

Received: 19 November 2019

Accepted: 07 January 2020

Published: 27 February 2020

Citation:

Shi M, Nie P, Fan Z, Fu R, Fang S, Dou $H$ and Zhang $X$ (2020) Tubular Graphene Nano-Scroll Coated Silicon for High Rate Performance Lithium-Ion Battery. Front. Energy Res. 8:2. doi: 10.3389/fenrg.2020.00002

\section{Tubular Graphene Nano-Scroll Coated Silicon for High Rate Performance Lithium-Ion Battery}

\author{
Minyuan Shi, Ping Nie, Zengjie Fan, Ruirui Fu, Shan Fang, Hui Dou and Xiaogang Zhang * \\ Jiangsu Key Laboratory of Electrochemical Energy Storage Technologies, College of Materials Science and Engineering, \\ Nanjing University of Aeronautics and Astronautics, Nanjing, China
}

Owing to its high specific capacity and abundant reserves, silicon has been considered as a promising anode material for future lithium storage. However, its cycle life is seriously hindered by the huge volume variation and low conductivity. Therefore, it is crucial to effectively and easily prepare silicon anode with excellent performance. Herein, we introduce a simple method to prepare graphene nano-scroll coated silicon nanoparticles (Si@rGONSs) on a large scale. With the open tubular structure and the high conductivity of graphene, the obtained Si@rGONSs-2 electrode delivers a superior rate capacity of $866 \mathrm{mAh} \mathrm{g}^{-1}$ at $5 \mathrm{~A} \mathrm{~g}^{-1}$ and a capacity retention of $89.4 \%$ after 300 cycles. Furthermore, the Si@rGONSs//Li[ $\left[\mathrm{Ni}_{1 / 3} \mathrm{Co}_{1 / 3} \mathrm{Mn}_{1 / 3}\right]_{2}$ (NCM 111) full cell was successfully demonstrated, which achieves a stable capacity of $128.5 \mathrm{mAh} \mathrm{g}^{-1}$ at $0.5 \mathrm{C}$ $\left(1 \mathrm{C}=185 \mathrm{mAh} \mathrm{g}^{-1}\right)$ and a high energy density of $406 \mathrm{Wh} \mathrm{kg}^{-1}$.

\footnotetext{
Keywords: silicon anodes, lithium-ion battery, three-dimensional graphene, graphene nano-scroll, high energy density
}

\section{INTRODUCTION}

Driven by the rapid development of power tools, electric vehicles, and portable electric devices, the development of next-generation rechargeable lithium-ion batteries (LIBs) with both high energy density and high power density becomes a global priority (Balogun et al., 2016; Rahman et al., 2016; Zhang et al., 2018). Unfortunately, the current commercial graphite-based anode only delivers a limited theoretical capacity $\left(372 \mathrm{mAh} \mathrm{g}^{-1}\right.$ ), which couldn't meet the increasing demands (Kim et al., 2012). Therefore, it is imminent to search new anode materials with higher specific capacity. Owing to the ultrahigh theoretical capacity $\left(4,200 \mathrm{mAh} \mathrm{g}^{-1}\right)$, environmental friendliness, low cost, non-toxicity, and suitable Li-uptake voltage, silicon has been considered to be one of the strong candidates for next-generation lithium battery anodes (Chae et al., 2019). However, two intrinsic obstacles severely restrict its practical applications: (a) the huge volume variation during cycling (400\%), which brings about the active substances that fall off from the current collector and continuously generated solid electrolyte interphase (SEI); and (b) poor electrical and ionic conductivity, which results in hysteresis in electrochemical kinetics and poor rate performance (Tao et al., 2019).

Many methods have been proposed to address the above problems. Combining silicon with conductive carbon matrix is an effective method, which could not only relieve the huge volume expansion of silicon but also improve the electron conductivity of the composite (Yue et al., 2018). Owing to the superior chemical stability, excellent mechanical flexibility, and high electron conductivity of graphene, preparation of graphene/electroactive material composites have been proved to be an effective way to improve the cycling stability and rate performance of composites 


\section{Freeze drying \\ Si NPs/Go solution Si NPs@GONSs

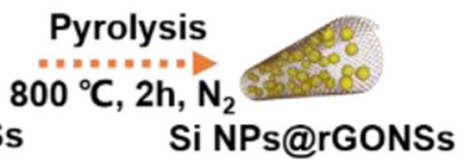

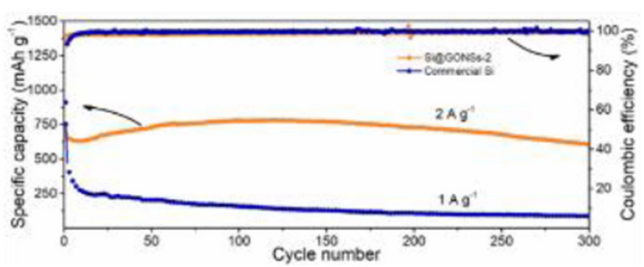

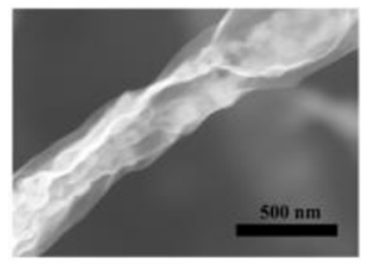

GRAPHICAL ABSTRACT | Tubular graphene nano-scroll coated silicon composite enhanced lithium storage performance.

(Kamali et al., 2017). However, silicon/graphene composites with simple mixing couldn't effectively improve the cycling performance and rate performance of the anode, due to low contact area between silicon nanoparticles and graphene sheets, poor encapsulation efficiency, and graphene sheets tending to agglomerate (Liu et al., 2017).

Three-dimensional (3D) graphene materials, which are prepared with appropriate assembly methods from twodimensional graphene sheets (Li et al., 2019a), not only maintain the advantages of two-dimensional graphene sheets but also possess superior structural properties, such as interconnected conductive network and without stacking (Luo et al., 2011). Therefore, preparing Si@3D graphene composite is a very good choice for achieving LIB anode with superior rate ability, high capacity, and excellent cycling stability (Dong et al., 2012). Nowadays, various Si@3D graphene structures have been synthesized, such as silicon/graphene balls (Yan et al., 2019), silicon/graphene fibers (Wang et al., 2010), silicon/graphene tubes (Wang et al., 2017), silicon/vertical graphene composites (Viculis et al., 2003), and other 3D graphene architectures (Chen et al., 2011; Zhai et al., 2017). In order to obtain the above structures, various preparation methods have been employed, such as self-assembly methods (Zhang et al., 2017), chemical vapor deposition (CVD) methods (Tang et al., 2019), and other methods (Luo et al., 2013; Wang et al., 2014). However, most synthetic methods are expensive or time consuming, so there is still a need for viable techniques for large-scale production of silicon @3D graphene materials with high quality and controllable structures (Zhou et al., 2015).

Graphene scroll, as one of 3D graphene structures, have been widely applied in various energy storage systems (Shin et al., 2014; Chen et al., 2018a), owing to its open tubular structure, which could provide fast electronic transmission channel, and interior space to contain volume expansion. We think that the tubular graphene scroll could be used on the silicon negative electrode. Herein, tubular graphene nano-scroll coated silicon (Si@rGONSs) composite has been compounded by a costeffective and non-toxic method, which is very easy to scale up. Owing to the tightly wrapped graphene and open tubular structure, the Si@rGONSs has a stable SEI and fast Li-ion transmission channels. Furthermore, the space between silicon particles in graphite tubular structure could adapt the volume variation of silicon negative electrode. Under the synergy of the above reasons, the obtained Si@rGONSs shows an outstanding cycle stability and superior rate performance for LIBs.

\section{EXPERIMENTAL SECTION}

\section{Material Synthesis}

Fifty milligrams of commercial silicon nanoparticles (80$100 \mathrm{~nm}$ ) and $5 \mathrm{mg}$ of polyvinylpyrrolidone were mixed with $50 \mathrm{ml}$ of $\mathrm{H}_{2} \mathrm{O}$ and then stirred overnight. Secondly, the $\mathrm{Si}$ aqueous dispersion $\left(1.0 \mathrm{mg} \mathrm{ml}^{-1}\right.$ ) was mixed with the GO suspension $\left(1.0 \mathrm{mg} \mathrm{ml}^{-1}, 50 \mathrm{ml}\right.$ ) and was stirred for $12 \mathrm{~h}$. Subsequently, the mixture was heated to $90^{\circ} \mathrm{C}$ and then put into liquid nitrogen quickly. The completely frozen solid was freeze dried to remove water and then reduced by thermal treatment at $800^{\circ} \mathrm{C}$ in argon (Ar) atmosphere for $2 \mathrm{~h}$. The Si@rGONSs could be obtained after thermal treatment at $800^{\circ} \mathrm{C}$ for $2 \mathrm{~h}$ in Ar atmosphere. With changed $\mathrm{Si}$ and GO weight ratio (Si:GO), 2:1, 1:1, and 1:2, the Si@rGONSs could be marked as Si@rGONSs-1, Si@rGONSs-2, and Si@rGONSs-3.

\section{Characterization}

The field emission scanning electron microscope (FESEM) images of the samples were recorded on a JEOL JSM-6380LV FESEM. The high-resolution transmission electron microscopy (HRTEM) and transmission electron microscopy (TEM) images were collected with JEOL JEM-2010 and JEOL JEM-2100, respectively. Raman spectra were recorded by the HORIBA Scientific Lab RAM HR Raman spectrometer system with an excitation wavelength of $532.4 \mathrm{~nm}$. The crystalline phases were measured via X-ray diffraction using $\mathrm{Cu} \mathrm{K} \alpha(\lambda=0.154178 \mathrm{~nm})$. The XPS was performed using an ESCALAB 250Xi spectrometer with a mono $\mathrm{Al} \mathrm{K} \alpha$ radiation. The carbon content of the sample was determined using thermogravimetric analysis (TGA, NETZSCH STA4094C).

\section{Electrochemical Measurements}

The working electrodes were prepared by pasting a slurry with active materials (80 wt\%), sodium carboxymethyl cellulose (CMC, 10 wt $\%)$, and acetylene black (10 wt\%) with water 
onto a copper foil, and vacuum dried at $60^{\circ} \mathrm{C}$ overnight. The electrolyte is $1 \mathrm{M} \mathrm{LiPF}_{6}$ in ethylene carbonate/diethyl carbonate (EC/DMC, 1:1 by vol) with 5 vol\% fluoroethylene carbonate (FEC) as electrolyte. The $\mathrm{Li}\left[\mathrm{Ni}_{1 / 3} \mathrm{Co}_{1 / 3} \mathrm{Mn}_{1 / 3}\right] \mathrm{O}_{2}$ cathodes were prepared with commercial $\mathrm{Li}\left[\mathrm{Ni}_{1 / 3} \mathrm{Co}_{1 / 3} \mathrm{Mn}_{1 / 3}\right] \mathrm{O}_{2}$ powders (80 wt \%), PVDF (10 wt \%), and acetylene black (10 wt\%) in N-methyl-2-pyrrolidone (NMP) with a weight ratio of $8: 1: 1$. The half and full cell tests were conducted by using the 2032 coin cell type at room temperature. Before assembling the full batteries, Si@GONSs-2 anodes were electrochemically pre-lithiated by direct contacting with a lithium foil for $30 \mathrm{~min}$ to compensate for the initial active lithium loss that comes from electrolyte and cathode material. The battery was cathode limited, because excess silicon could prevent the lithium deposition during charging. The capacity ratio of the negative electrode to the positive electrode is approximately 1.2-1.5:1. After cycling, the batteries were disassembled in the glove box and washed with methanol and dimethyl carbonate solution several times for morphology characterizations.

The energy density value was calculated from the full cell based on the weight of electrode materials without considering the weight of the electrolyte, current collector, separator, and case in cells configuration. Galvanostatic cycling tests were carried out on a Land Battery Test System (Wuhan LAND electronics Co., Ltd., China). The electrochemical impedance spectroscopy (EIS) and cyclic voltammetry (CV) were tested with CHI 760E electrochemical workstations.

\section{RESULTS AND DISCUSSIONS}

The fabrication process for Si@rGONSs composites is shown in Figure 1A. With simple nitrogen freezing and high-temperature reduction, the Si@rGONSs composites could be obtained. Si@rGONSs composites with different Si and graphene ratio of
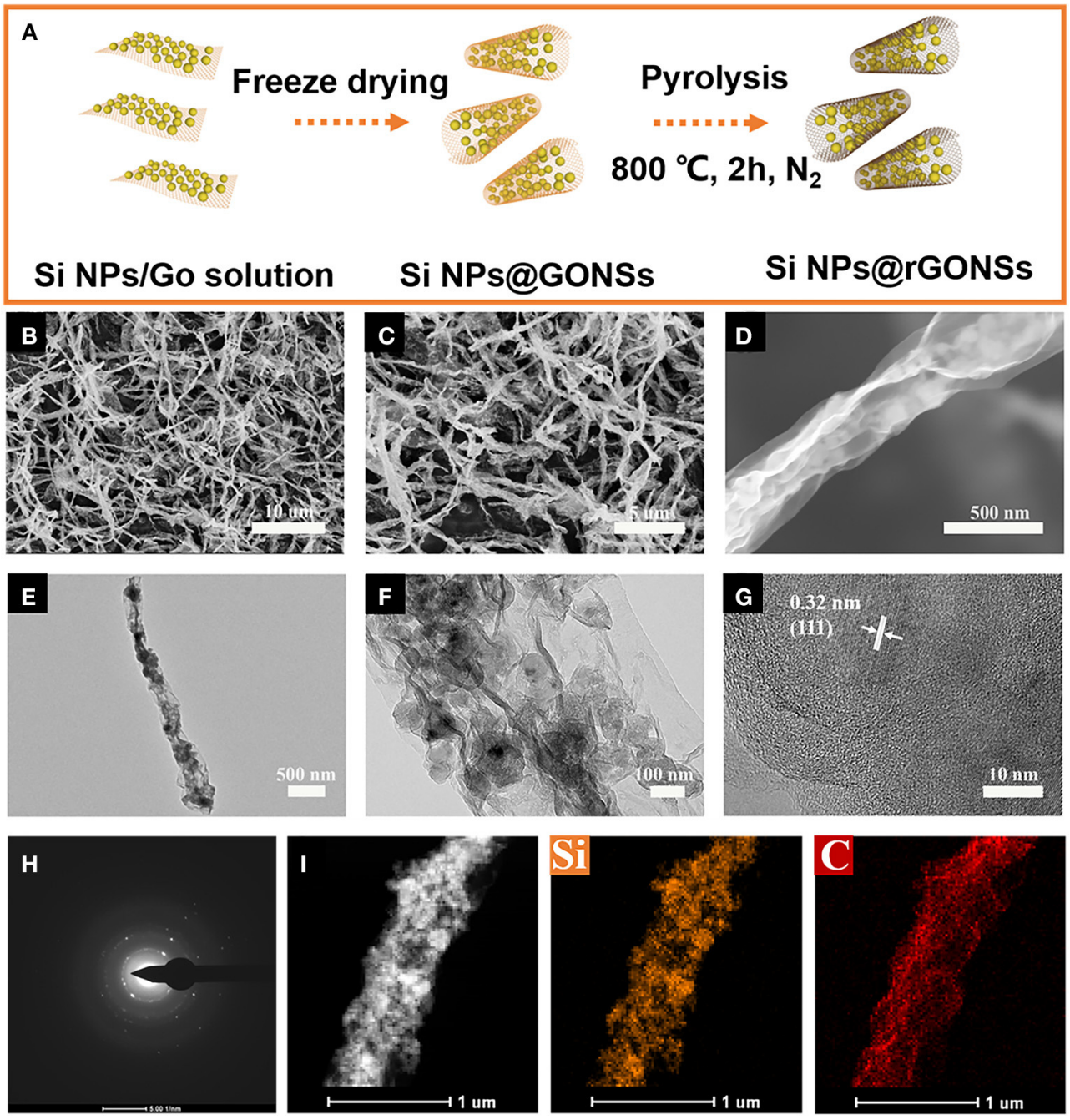

FIGURE 1 | (A) Schematic fabrication process of Si@rGONSs-2, (B,C,D) SEM images, (E,F) TEM images, (G) HRTEM image, (H) SAED image, and (I) elemental mapping results of Si@rGONSs-2. 
2:1, 1:1, and 1:2 are denoted as Si@rGONSs-1, Si@rGONSs2, and Si@rGONSs-3, respectively. The morphology and structure of Si@rGONSs-2 are investigated by SEM and TEM. In Figures 1B-D, tubular graphene coated silicon can be clearly observed with a length about $7-8 \mu \mathrm{m}$. The uncalcined Si@rGONSs-2 is shown in Figures S1A,B, which indicates that, during the reduction process, the morphology of Si@rGONSs2 is well-maintained. High-magnification transmission electron microscopy images (Figure 1E) further confirm the tubular structure; meanwhile, silicon nanoparticles are evenly wrapped inside the graphene scroll. The size of silicon particles is about $80 \mathrm{~nm}$ and there is enough space between silicon particles that could accommodate the volume variation of the silicon anode (Figure 1F). TEM images of Si@rGONSs-1 and Si@rGONSs-3 are shown in Figures S2A,B, and it is obvious that Si@rGONSs composites with higher silicon ratio have more silicon particles in one graphene scroll. In addition, the 0.32-nm lattice spacing of $\mathrm{Si}$ and three coaxial diffraction rings belonging to the $\mathrm{Si}$ (111), (220), and (311) planes could be observed in Figures 1G,H, revealing its polycrystalline structure ( $\mathrm{Li}$ et al., 2019b). The elemental distribution of Si@rGONSs-2 is further examined by X-ray (EDX) elemental mappings (Figure 1I), which clearly illustrated the uniform distribution and tight contact of Si nanospheres in the graphene scroll (Chen et al., 2018b).

To confirm the compositional information and structure properties of Si@rGONSs composites, XRD, Raman spectroscopy, and XPS were performed. All composites have the same five peaks in the XRD patterns (Figure 2A), which can be readily directed to crystalline silicon, suggesting that the properties of crystalline silicon are maintained during the preparation processes (Li et al., 2018). At the same time, all the Si@rGONSs composites have an additional broad peak around $25^{\circ}$, which could be confirmed to the rGO (Agyeman et al., 2016). The Raman spectra (Figure 2B) shows typical silicon peaks (520 and $945 \mathrm{~cm}^{-1}$ ) and two typical carbon peaks corresponding to the carbon $\mathrm{D}$ and $\mathrm{G}$ bands $\left(1,250\right.$ and $\left.1,450 \mathrm{~cm}^{-1}\right)$. The intensity ratio of the $\mathrm{D}$ band peak to the $\mathrm{G}$ band peak is about 0.92 in all the Si@rGONSs composites; it indicates that the graphene scroll has a few defects, which could be part of oxygen moieties remaining after calcination (Kamali et al., 2017). TGA was used to quantify the mass percentage of graphene in Si@rGONSs-2. As presented in Figure 2C, the Si@rGONSs-2 has a large mass loss of 25\%, corresponding to the decomposition of graphene matrix in the sample. So, the overall percentage of Si in Si@rGONSs-2 could be easily determined to be about $75 \%$. Peaks shown using X-ray photoelectron spectroscopy (XPS) could be attributed to carbon and silicon (Figure 2D); the Si peaks are much lower than the peak of carbon because the silicon is completely wrapped by the graphene scroll. In the case of the high-resolution XPS spectra, C 1s (Figure 2E), peaks fitted at $\sim 284.1, \sim 285.4$, and $\sim 286.2 \mathrm{eV}$ are successively assigned to graphene surface functional groups of $\mathrm{C}-\mathrm{C}, \mathrm{C}=\mathrm{C}$, and $\mathrm{C}-\mathrm{O}$, respectively (Song et al., 2018). For the XPS spectrum, Si 2 p could be derived from two peaks located at 99.5 and $104 \mathrm{eV}$ (Figure 2F), which could be attributed to $\mathrm{Si}$ and $\mathrm{SiO}_{2}$, respectively ( $\mathrm{He}$ et al., 2018). Owing to the surface of silicon particles having an oxide layer of several nanometers
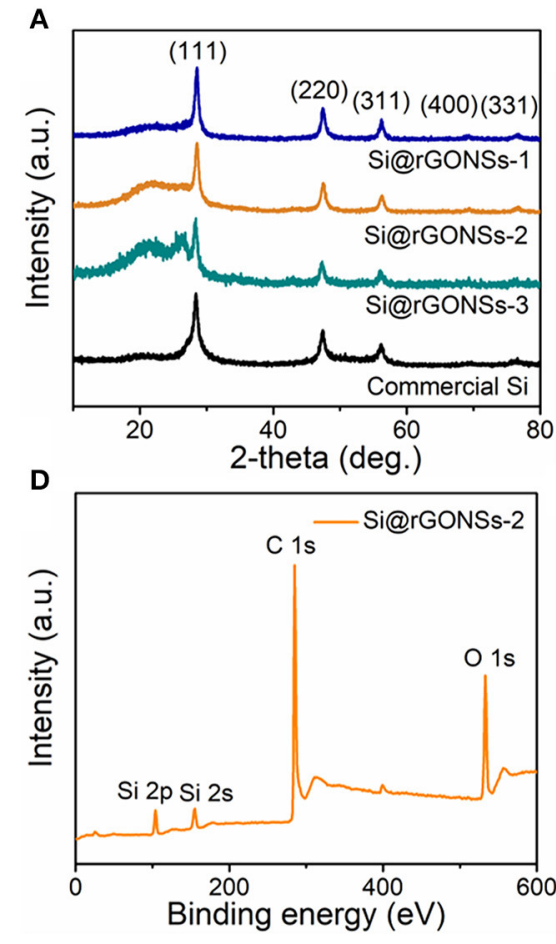
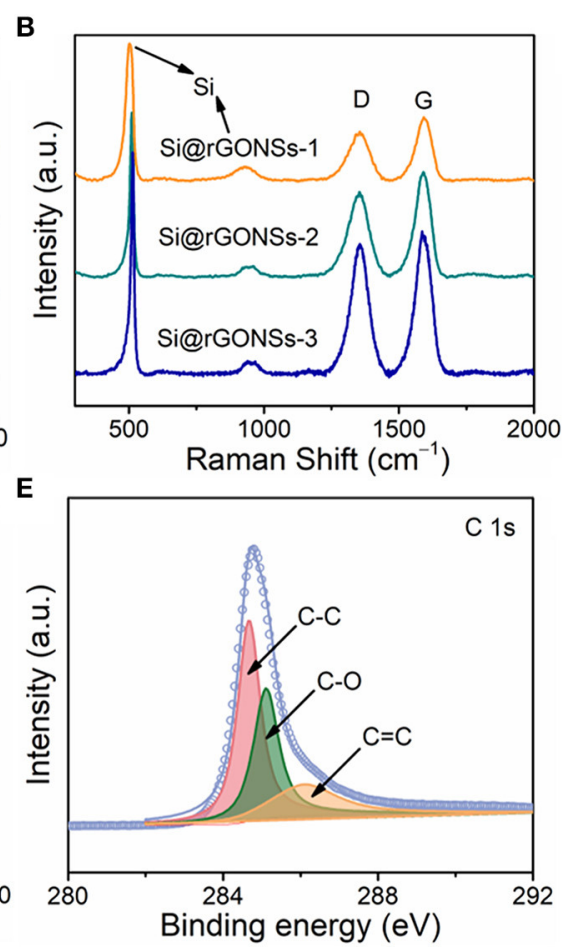

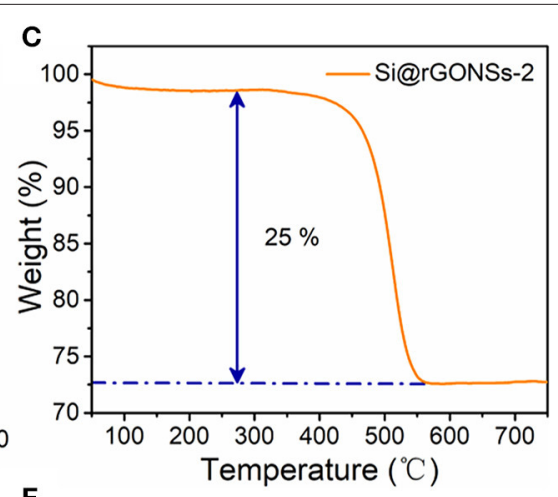

$\mathbf{F}$

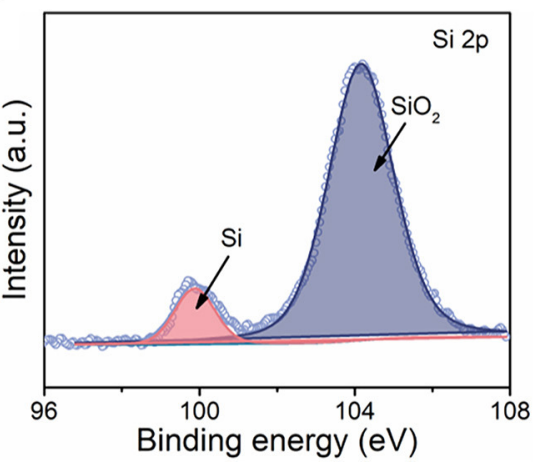

FIGURE 2 | (A) XRD patterns and (B) Raman spectrum of Si@rGONS composites, (C) thermogravimetric analysis (TGA) data of Si@rGONSs-2, (D) XPS survey spectrum of Si@rGONSs-2, and (E,F) high-resolution C 1s and Si 2p spectrum of Si@rGONSs-2. 

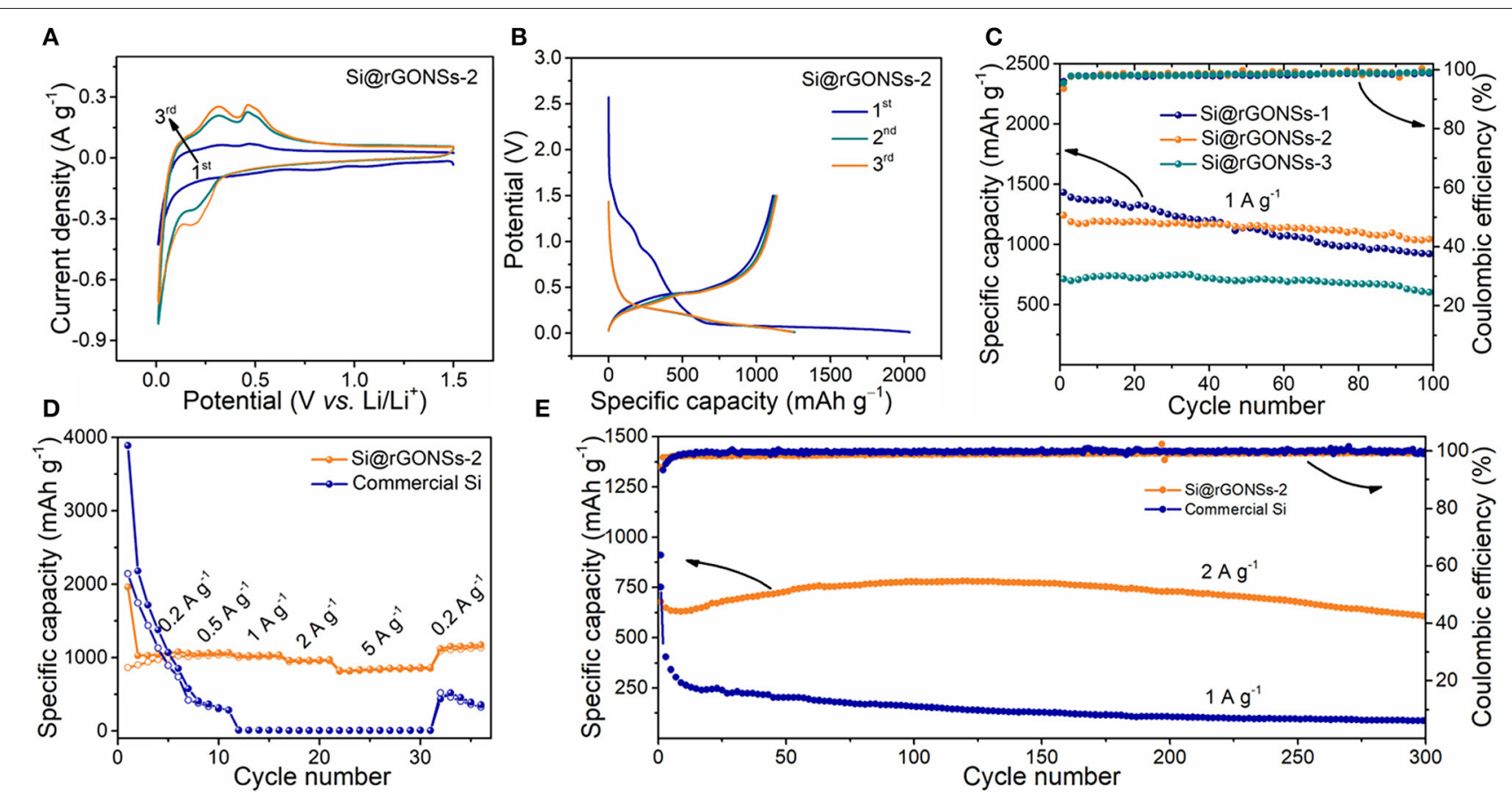

FIGURE 3 | Electrochemical performances of Si@rGONS composites. (A) CV curves at a scan rate of 0.1 mV s ${ }^{-1}$ of Si@rGONSs-2, (B) first three galvanostatic discharge-charge curves at 200 mA g $^{-1}$ of Si@rGONSs-2, (C) cycling curves of Si@rGONS composites at 1 A g $\mathrm{g}^{-1}$, (D) rate curves of Si@rGONSs-2 and commercial $\mathrm{Si}$, and (E) cycling curves of Si@rGONSs-2 at $2 \mathrm{~A} \mathrm{~g}^{-1}$ and commercial Si at $1 \mathrm{~A} \mathrm{~g}^{-1}$.

thick naturally and the detection depth of XPS being just a few nanometers, the content of silica is higher than the silicon in the XPS test (Li et al., 2018).

The electrochemical properties of Si@rGONSs composites were investigated by building up CR 2032 coin cells with the lithium foil as counter and reference electrode at room temperature. The CV curves were adopted to investigate the lithium-ion insertion/deinsertion phenomenon of Si@rGONSs2 at $0.1 \mathrm{mV} \mathrm{s}^{-1}$ between 0.01 and $1.5 \mathrm{~V}$ with the first three cycles (Figure 3A) (Nie et al., 2017). In the first cathodic sweep, the broad irreversible curve located at $0.6-1.2 \mathrm{~V}$ could be attributed to the SEI formation. Because of the silicon changes from crystal to amorphous after the first delithiation, the peak located below $0.1 \mathrm{~V}$ during the first cycle is replaced by the peak at about $0.25 \mathrm{~V}$ during the subsequent cycling. In the anodic scan, two oxidation peaks appearing at around 0.35 and $0.53 \mathrm{~V}$ could be related to the dealloying process of LixSi. Compared with the commercial silicon (Figure S3A), the Si@rGONSs-2 shows smaller polarization because of the closer position between anodic and cathodic peaks, implying its faster lithiation/delithiation kinetics. Figure 3B shows galvanostatic discharge/charge profiles of the Si@rGONSs-2 electrode in the first three cycles between 0.01 and $1.5 \mathrm{~V}$ at $0.2 \mathrm{~A} \mathrm{~g}^{-1}$ (Wang et al., 2018). During the first discharge process, there is a loping plateau between 0.1 and $1.2 \mathrm{~V}$, which could correspond to the formation of SEI. The flat plateau at about $0.1 \mathrm{~V}$ could index to the lithiation of the crystal silicon.

The Si@rGONSs-2 electrode delivers a large initial charge capacity of $1112.6 \mathrm{mAh} \mathrm{g}^{-1}$ with the initial coulombic efficiency (ICE) of $54.6 \%$. In contrast, commercial Si has an ICE of
$52.8 \%$ with a $4,201 \mathrm{mAh}^{-1}$ high initial discharge capacity in the first cycle, but after three cycles, only 1,677 mAh $\mathrm{g}^{-1}$ capacity retained (Figure S3B). The Si@rGONSs-1 delivers an initial discharge capacity of $3,540 \mathrm{mAh} \mathrm{g}^{-1}$ and the ICE is 66.4\% (Figure S4B). The Si@rGONSs-3 delivers an initial discharge capacity of $3,540 \mathrm{mAh}^{-1}$ and the ICE is $47.3 \%$ (Figure S4A). The pure graphene electrode has a low ICE of $42.7 \%$. The huge irreversibility in the ICE could be attributed to introducing graphene, which would inevitably decrease the ICE (Figures S5A,B) (Nie et al., 2018).

The cycling stability performance comparison of different ratios of Si@rGONSs composites at $1 \mathrm{~A} \mathrm{~g}^{-1}$ is given in Figure 3C. It can be found that the Si@rGONSs-2 anode retains a stable capacity of $1,038.2 \mathrm{mAh} \mathrm{g}^{-1}$ after 100 cycles, which is larger than Si@rGONSs-3 (598.5 mAh $\mathrm{g}^{-1}$ ). The Si@rGONSs-1 has the highest initial capacity of $1,430.1 \mathrm{mAh}$ $\mathrm{g}^{-1}$; however, the capacity only maintains $64 \%$ after 100 cycles. Therefore, we choose Si@rGONSs-2 as a suitable composite to explore other electrochemical properties and compare it with the commercial silicon anode. Rate performance is another key point that affects practical applications. At current densities of $0.2,0.5,1,2$, and $5 \mathrm{~A} \mathrm{~g}^{-1}$, the Si@rGONSs-2 delivers reversible capacities of 1,062, 1,055, 1,026, 964, and $866 \mathrm{mAh} \mathrm{g}^{-1}$, respectively (Figure 3D). Impressively, when the charge/discharge density tunes back to $0.2 \mathrm{~A} \mathrm{~g}^{-1}$, the specific capacity of $1,126 \mathrm{mAh}^{-1}$ could recover, indicating its excellent rate performance. The slight enhancement in the specific capacity when the current density was reduced back to $0.2 \mathrm{~A} \mathrm{~g} \mathrm{~g}^{-1}$ is due to gradual activation. It is a very common phenomenon in silicon negative electrodes, which 
means that the capacity will slowly increase during the first several cycles (Li et al., 2019b). On the other hand, the commercial Si delivers a grand initial capacity of $2,181 \mathrm{mAh} \mathrm{g}^{-1}$, but it could not withstand high current density. Excellent rate performance may result from the superior electronic conductive framework and the open tubular structure for ion transport. Due to the fast charging/discharging performance, we further evaluate the long-term performance of the composite at $2 \mathrm{~A}$ $\mathrm{g}^{-1}$. The Si@rGONSs-2 anode demonstrates an 89.4\% capacity retention after 300 cycles, with $99.9 \%$ coulombic efficiency (Figure 3E). The excellent rate performance and superior cycling stability could be ascribed to the uniform coating of graphene scroll, which could offer high conductivity and fast ion transmission channel.

In order to further understand the origin of the excellent electrochemical performance of the Si@rGONSs-2 composite, a series of tests were carried out. The electrochemical impedance spectrum (EIS) of Si@rGONSs-2 and commercial Si is compared to investigate the charge transport kinetics (Figure S7). The semicircle in high frequency and the sloping line in the low-frequency region are assigned to the charge transfer resistance $\left(R_{\mathrm{ct}}\right)$ on the electrode-electrolyte interface and lithium-ion diffusion behavior in the electrode (Figure 4A). The Si@rGONSs-2 electrode shows a smaller $R_{\mathrm{ct}}$ than commercial $\mathrm{Si}$, which means a lower charge-transfer impedance (Fang et al., 2014). In the Nyquist plot, the first semicircle in the highfrequency region after cycling represents the ohmic resistance of the SEI layer, which could evaluate the stability of the SEI film (Zhang et al., 2019). As shown in Figure 4B, the $R_{\mathrm{f}}$ value of commercial silicon is much higher than the Si@rGONSs2 electrode, which means during cycling, the SEI film of the Si@rGONSs-2 is much stable compared to commercial Si. The sloping line in the low-frequency region is related to lithium-ion diffusion behavior in the electrode. As shown in Figure 4C, the lower slope of the Si@rGONSs-2 composite electrode indicates that the lithium-ion diffusion kinetics inside the electrode is stable and easy. Moreover, the CV curves at various scan rates are
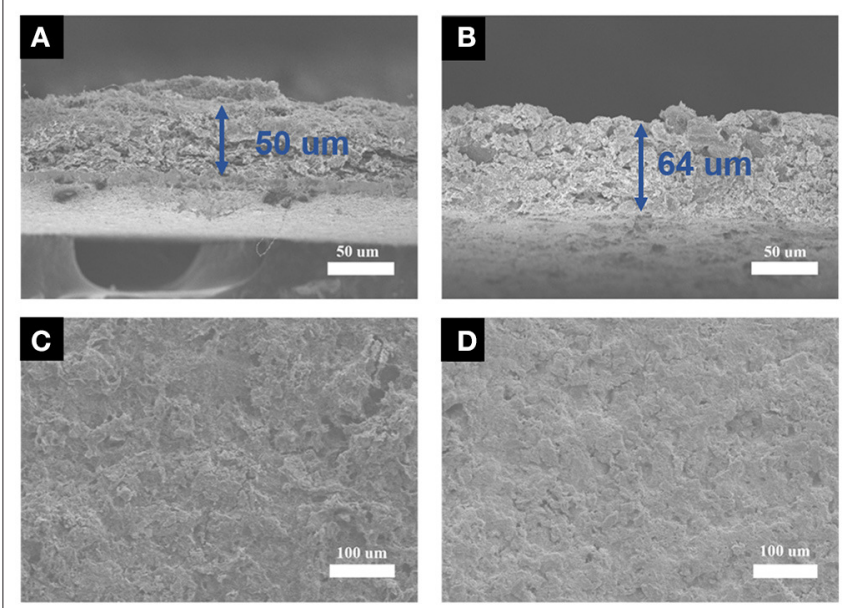

FIGURE 5 | Electrode thickness of Si@rGONSs-2 electrode before cycling (A) and after 100 cycles, (B) SEM images of Si@rGONSs-2 electrode, (C) before cycling, and (D) after 100 cycles.
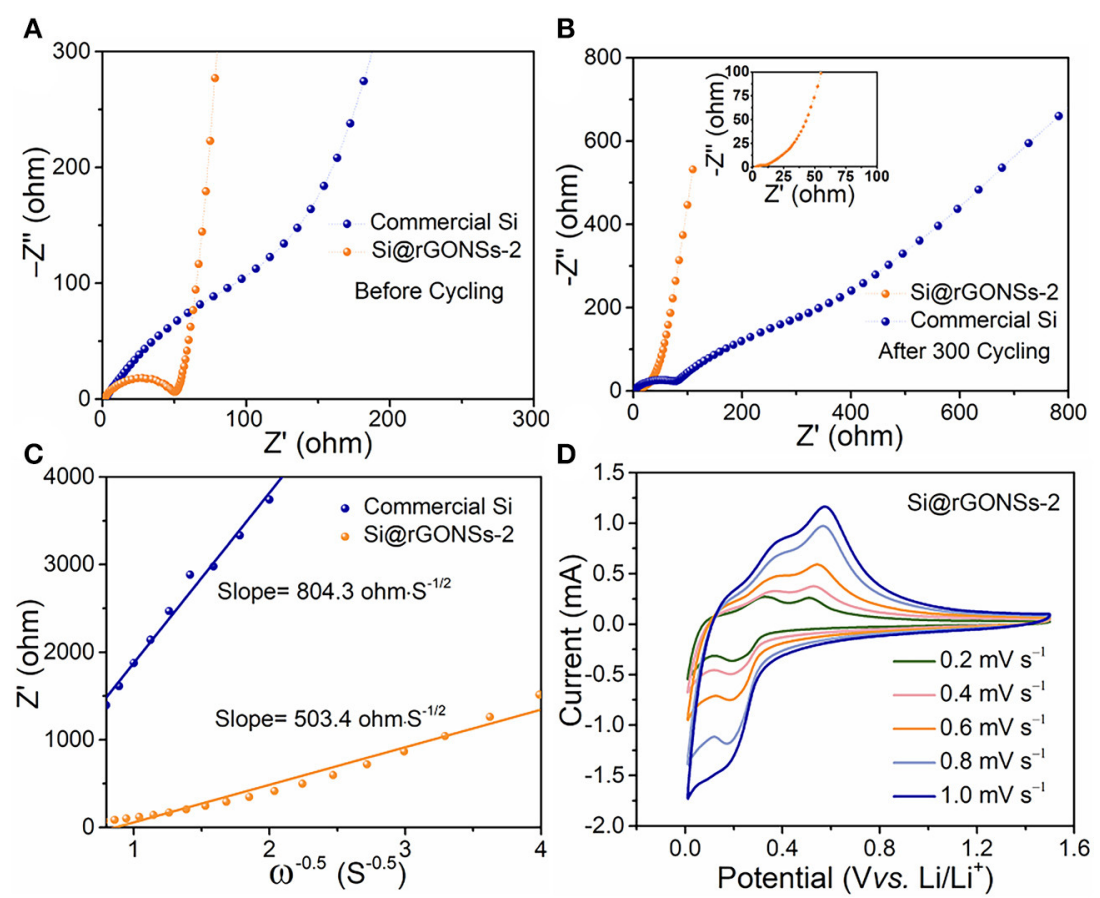

FIGURE 4 | (A) Nyquist plots of the pure silicon and Si@rGONSs-2 in fresh state, (B) Nyquist plots of the pure silicon and Si@rGONSs-2 after 300 cycles, (C) the corresponding liner fits (relationship between $Z^{\prime}$ and $\omega^{-1 / 2}$ ) in low-frequency region derived from (A), and (D) CV profiles of the Si@rGONSs-2 at scan rates from 0.2 to $1 \mathrm{mV} \mathrm{s}^{-1}$. 
A

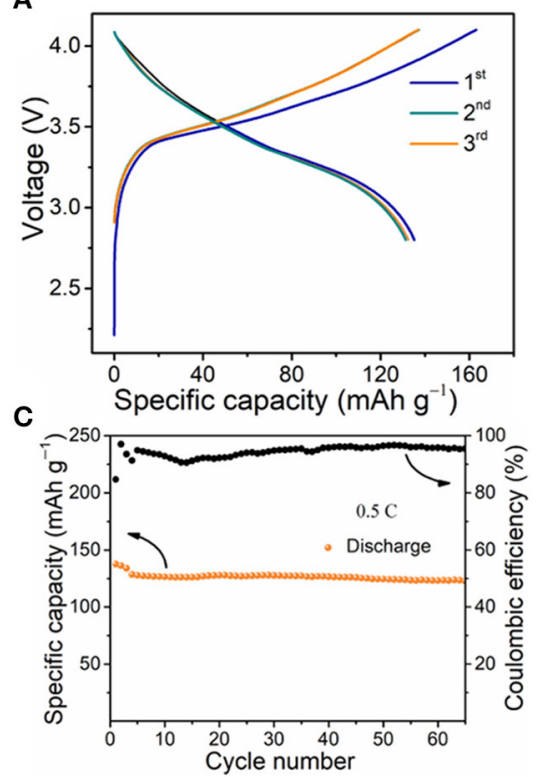

B

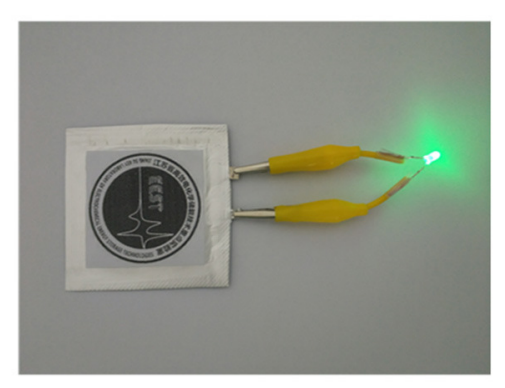

D

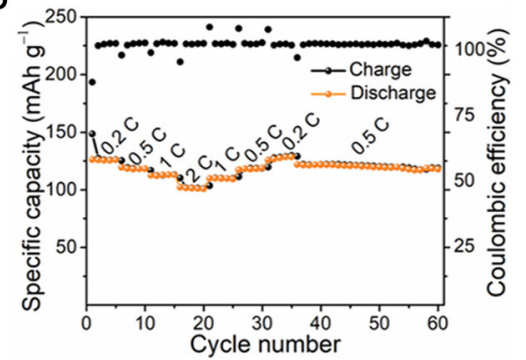

FIGURE 6 | Electrochemical performances of full-cell. (A) Charge-discharge profiles for a Si@rGONSs-2//Li[ $\left.\mathrm{Ni}_{1 / 3} \mathrm{Co}_{1 / 3} \mathrm{Mn}_{1 / 3}\right] \mathrm{O}_{2}$ (NCM 111) full cell between 2.8 and 4.1 V at 0.5 C, (B) the photograph of LED lamps lighted by Si@rGONSs-2//NCM 111 pouch cell, (C) cycling performance of Si@rGONSs-2//NCM 111 full cell at $0.5 \mathrm{C}$, and $(\mathbf{D})$ rate performance from 0.2 to $2 \mathrm{C}$.

a strong evidence for verifying the rate performance. As shown in Figure 4D, the Si@rGONSs-2 displays similar redox reaction shapes, which means a good response to the electrochemical ability of Si@rGONSs-2 to different scan rates and implies a superior rate performance (Sun et al., 2016).

The thickness change of the electrode before and after the cycle can reflect the degree of the electrode deformation; the Si@rGONSs-2 electrode only has a low swelling rate of $28 \%$, which demonstrates a small volume change of the composite material (Figures 5A,B). The one-dimensional tubular morphology of Si@rGONSs-2 could be well-preserved after 100 cycles (Figure S8), indicating that the unique graphene scroll structure and suitable pores could effectively accommodate the huge volume variation during circulation and maintain the structural stability of the composite. As shown in Figures 5C,D, the Si@rGONSs-2 electrode after 100 cycles retains its original appearance with no crack or peel-off phenomenon (Xu et al., 2018). The contact angles of commercial Si and Si@rGONSs2 electrode are 20.2 and $9.5^{\circ}$, respectively (Figures S6A,B), which shows that the Si@rGONSs-2 electrode has better electrolyte wetting performance compared with commercial $\mathrm{Si}$ (Song et al., 2018). Better electrolyte wetting and the open tubular structure provides space to handle variation in volume; thus, the Si@rGONSs-2 electrode possesses outstanding stability and reversibility.

To further explore the practicality of Si@rGONSs-2, full cells with NCM 111 as cathode are assembled. Because the ICE value of the Si@rGONSs-2 isn't very high, the anode was firstly prelithiated (Liu et al., 2018a). Owing to the substantial energy capacity, cycling stability, low cost, and wide operating voltage window, the NCM 111 cathode has become one of the most promising cathode materials (An et al., 2019). As shown in
Figures S9A-D, the NCM 111 half cell has a high initial capacity of $120.2 \mathrm{mAh} \mathrm{g}^{-1}$ after 100 cycles, the capacity retention is $93.6 \%$ at $1 \mathrm{C}$ between 3.0 and $4.2 \mathrm{~V}$. When assembled into a full cell, obvious discharge plateaus between 3.3 and $4.1 \mathrm{~V}$ can be observed (Figure 6A). At the same time, the LED could be successfully lighted by the pouch cell (Figure 6B). In addition, the Si@rGONSs-2//NCM 111 full cell demonstrates a stable cycling performance at $0.5 \mathrm{C}$ (Figure 6C); after 65 cycles, 89\% initial capacity could be maintained. The Si@rGONSs-2//NCM 111 full cell delivers an energy density of $409 \mathrm{Wh} \mathrm{kg}^{-1}$, which is much higher than the state-of-the-art batteries (about $170 \mathrm{Wh} \mathrm{kg}^{-1}$; Liu et al., 2018b). Furthermore, the Si@rGONSs-2//NCM 111 full cell also has superior rate ability; the specific capacities are 126 and $102 \mathrm{mAh} \mathrm{g}^{-1}$ at 0.2 and $2 \mathrm{C}$, respectively. Even after the cycling test at different current densities, the reversible capacity of the Si@rGONSs-2//NCM 111 full cell can still be well-maintained at $120 \mathrm{mAh} \mathrm{g}^{-1}$, indicating the outstanding rate performance and cycling stability (Figure 6D).

\section{CONCLUSION}

This work fabricated a graphene scroll coated nano-silicon as anodes; meanwhile, the reasons for the superior electrochemical performance and the feasibility for full cell assembly are explored. The one-dimensional Si@rGONSs cross-link each other externally and silicon particles are tightly attached to graphene internally, and the whole electrode has a superior conductive network. Moreover, the pores between the silicon particles provide sufficient space for volume expansion. With these extraordinary features, the achieved Si@rGONSs-2 anode exhibits superior rate capability and excellent cycling performance. Furthermore, the Si@rGONSs-2 anode also 
demonstrates superior electrochemical performance when assembled into a full battery. After 65 cycles, the full cell still delivers $409 \mathrm{Wh} \mathrm{kg}^{-1}$ high energy density with a high coulombic efficiency. Owing to its rational design, easy scale-up method, and attractive electrochemical properties, Si@rGONSs-2 provides a means to obtain stable silicon anodes with long cycle life and superior rate performance for high-energy lithium-ion batteries.

\section{DATA AVAILABILITY STATEMENT}

All datasets generated for this study are included in the article/Supplementary Material.

\section{AUTHOR CONTRIBUTIONS}

All authors listed have made a substantial, direct and intellectual contribution to the work, and approved it for publication.

\section{REFERENCES}

Agyeman, D., Song, K., Lee, G., Park, M., and Kang, Y. (2016). Carbon-coated Si nanoparticles anchored between reduced graphene oxides as an extremely reversible anode material for high energy-density Li-ion battery. Adv. Energy Mater. 6:1600904. doi: 10.1002/aenm.201600904

An, W., Gao, B., Mei, S., Xiang, B., Fu, J., Wang, L., et al. (2019). Scalable synthesis of ant-nest-like bulk porous silicon for high-performance lithium-ion battery anodes. Nat. Commun. 10:1447. doi: 10.1038/s41467-019-09510-5

Balogun, M. S., Qiu, W., Luo, Y., Meng, H., Mai, W., Onasanya, A., et al. (2016). A review of the development of full cell lithium-ion batteries: the impact of nanostructured anode materials. Nano Res. 9, 2823-2851. doi: 10.1007/s12274-016-1171-1

Chae, S., Choi, S. H., Kim, N., Sung, J., and Cho, J. (2019). Integration of graphite and silicon anodes for the commercialization of high-energy lithium-ion batteries. Angew. Chem. Int. Ed. Engl. 59, 110-135. doi: 10.1002/anie.201902085

Chen, K., Cao, J., Lu, Q., Wang, Q., Yao, M., Han, M., et al. (2018a). Sulfur nanoparticles encapsulated in reduced graphene oxide nanotubes for flexible lithium-sulfur batteries. Nano Res. 11, 1345-1357. doi: 10.1007/s12274-017-1749-2

Chen, M., Li, B., Liu, X., Zhou, L., Yao, L., Zai, J., et al. (2018b). Borondoped porous Si anode materials with high initial coulombic efficiency and long cycling stability. J. Mater. Chem. A. 6, 3022-3027. doi: 10.1039/C7TA1 $0153 \mathrm{H}$

Chen, Z., Ren, W., Gao, L., Liu, B., Pei, S., and Cheng, H. M. (2011). Three-dimensional flexible and conductive interconnected graphene networks grown by chemical vapour deposition. Nat. Mater. 10, 424-428. doi: $10.1038 /$ nmat3001

Dong, Z., Jiang, C., Cheng, H., Zhao, Y., Shi, G., Jiang, L., et al. (2012). Facile fabrication of light, flexible and multifunctional graphene fibers. Adv. Mater. 24, 1856-1861. doi: 10.1002/adma.201200170

Fang, S., Shen, L., Xu, G., Nie, P., Wang, J., Dou, H., et al. (2014). Rational design of void-involved Si@TiO2 nanospheres as high-performance anode material for lithium-ion batteries. ACS Appl. Mater. Interfaces 6, 6497-6503. doi: 10.1021/am500066j

He, Y., Xu, G., Wang, C., Xu, L., and Zhang, K. (2018). Horsetail-derived Si@Ndoped carbon as low-cost and long cycle life anode for Li-ion half/full cells. Electrochim. Acta. 264, 173-182. doi: 10.1016/j.electacta.2018.01.088

Kamali, A. R., Kim, H.-K., Kim, K.-B., Vasant Kumar, R., and Fray, D. J. (2017). Large scale green production of ultra-high capacity anode consisting of graphene encapsulated silicon nanoparticles. J. Mater. Chem. A. 5, 19126-19135. doi: 10.1039/C7TA04335J

\section{FUNDING}

This work was supported by the National Natural Science Foundation of China (51672128, 21773118, 21875107, and 51802154); the Prospective Joint Research Project of Cooperative Innovation Fund of Jiangsu Province (BE2018122); the Natural Science Foundation of Jiangsu Province (BK20170778); the Priority Academic Program Development of Jiangsu Higher Education Institutions (PAPD); and the Foundation of Graduation Innovation Center in NUAA (kfjj20180613).

\section{SUPPLEMENTARY MATERIAL}

The Supplementary Material for this article can be found online at: https://www.frontiersin.org/articles/10.3389/fenrg. 2020.00002/full\#supplementary-material

Kim, T., Park, J., Chang, S., Choi, S., Ryu, J., and Song, H. (2012). The current move of lithium ion batteries towards the next phase. Adv. Energy Mater. 2, 860-872. doi: 10.1002/aenm.201200028

Li, G., Huang, B., Pan, Z., Su, X., Shao, Z., and An, L. (2019a). Advances in three-dimensional graphene-based materials: configurations, preparation and application in secondary metal ( $\mathrm{Li}, \mathrm{Na}, \mathrm{K}, \mathrm{Mg}, \mathrm{Al}$ )-ion batteries. Energy Environ. Sci. 12, 2030-2053. doi: 10.1039/C8EE03014F

Li, J., Li, Z., Huang, W., Chen, L., Lv, F., Zou, M., et al. (2019b). A facile strategy to construct silver-modified, $\mathrm{ZnO}$-incorporated and carboncoated silicon/porous-carbon nanofibers with enhanced lithium torage. Small 15:e1900436. doi: 10.1002/smll.201900436

Li, Y., Liu, W., Long, Z., Xu, P., Sun, Y., Zhang, X., et al. (2018). Si@C microsphere composite with multiple buffer structures for high-performance lithium-ion battery anodes. Chem. Eur. J. 24, 12912-12919. doi: 10.1002/chem.201801417

Liu, Y., Hassan Siddique, A., Huang, H., Fang, Q., Deng, W., Zhou, X., et al. (2017). In situ preparation of $\mathrm{Fe}_{3} \mathrm{O}_{4}$ in a carbon hybrid of graphene nanoscrolls and carbon nanotubes as high performance anode material for lithium-ion batteries. Nanotechnology 28:465401. doi: 10.1088/1361-6528/aa8dae

Liu, Z., Guan, D., Yu, Q., Xu, L., Zhuang, Z., Zhu, T., et al. (2018b). Monodisperse and homogeneous $\mathrm{SiO} / \mathrm{C}$ microspheres: a promising high-capacity and durable anode material for lithium-ion batteries. Energy Storage Mater. 13, 112-118. doi: 10.1016/j.ensm.2018.01.004

Liu, Z., Zhao, Y., He, R., Luo, W., Meng, J., Yu, Q., et al. (2018a). Yolk@Shell $\mathrm{SiO} / \mathrm{C}$ microspheres with semi-graphitic carbon coating on the exterior and interior surfaces for durable lithium storage. Energy Storage Mater. 19, 299-355. doi: 10.1016/j.ensm.2018.10.011

Luo, J., Jang, H., Huang, J., et al. (2013). Effect of sheet morphology on the scalability of graphene-based ultracapacitors. ACS Nano 7, 1464-1471. doi: $10.1021 / \mathrm{nn} 3052378$

Luo, J. Y., Dong, J. H., Tao, S., Li, X., Zhen, H., Katsoulidis, P. A., et al. (2011). Compression and aggregation resistant particles of crumpled soft sheets. ACS Nano 5, 8943-8949. doi: 10.1021/nn203115u

Nie, P., Le, Z., Chen, G., Liu, D., Liu, X., Wu, H. B., et al. (2018). Graphene caging silicon particles for high-performance lithium-ion batteries. Small 14:e1800635. doi: 10.1002/smll.201800635

Nie, P., Liu, X., Fu, R., Wu, Y., Jiang, J., Dou, H., et al. (2017). Mesoporous silicon anodes by using polybenzimidazole derived pyrrolic N-enriched carbon toward high-energy Li-ion batteries. ACS Energy Lett. 2, 1279-1287. doi: 10.1021/acsenergylett.7b00286

Rahman, M., Song, G., Bhatt, A., Wong, Y., and Wen, C. (2016). Nanostructured silicon anodes for high-performance lithium-ion batteries. Adv. Funct. Mater. 26, 647-678. doi: 10.1002/adfm.201502959 
Shin, Y. E., Sa, Y. J., Park, S., Lee, J., Shin, K. H., Joo, S. H., et al. (2014). An icetemplated, $\mathrm{pH}$-tunable self-assembly route to hierarchically porous graphene nanoscroll networks. Nanoscale 6, 9734-9741. doi: 10.1039/C4NR01988A

Song, M. S., Chang, G., Jung, D. W., Kwon, M. S., Li, P., Ku, J. H., et al. (2018). Strategy for boosting Li-ion current in silicon nanoparticles. ACS Energy Lett. 3, 2252-2258. doi: 10.1021/acsenergylett.8b01114

Sun, Z., Wang, X., Ying, H., Wang, G., and Han, W. Q. (2016). Facial synthesis of three-dimensional cross-linked cage for high-performance lithium storage. ACS Appl. Mater. Interfaces 8, 15279-15287. doi: 10.1021/acsami.6b02855

Tang, H., You, L., Liu, J., Wang, S., Wang, P., Feng, C., et al. (2019). Integrated polypyrrole@sulfur@graphene aerogel 3D architecture via advanced vapor polymerization for high-performance lithium-sulfur batteries. ACS Appl. Mater. Interfaces 11, 18448-18455. doi: 10.1021/acsami.9b04167

Tao, W., Wang, P., You, Y., Park, K., Wang, C., Li, Y., et al. (2019). Strategies for improving the storage performance of silicon-based anodes in lithium-ion batteries. Nano Res. 12, 1739-1749. doi: 10.1007/s12274-019-2361-4

Viculis, L. M., Mack, J. J., and Kaner, P. B. (2003). A chemical route to carbon nanoscrolls. Science 299, 1361-1361. doi: 10.1126/science.10 78842

Wang, B., Liu, Q., Han, J., Zhang, X., Wang, J., Li, Z., et al. (2014). Deft dipping combined with electrochemical reduction to obtain $3 \mathrm{D}$ electrochemical reduction graphene oxide and its applications in supercapacitors. J. Mater. Chem. A 2, 1137-1143. doi: 10.1039/C3TA13813E

Wang, J., Liao, L., Li, Y., Zhao, J., Shi, F., Yan, K., et al. (2018). Shellprotective secondary silicon nanostructures as pressure-resistant highvolumetric-capacity anodes for lithium-ion batteries. Nano Lett. 18, 7060-7065. doi: 10.1021/acs.nanolett.8b03065

Wang, R., Hao, Y., Wang, Z., Gong, H., and Thong, J. T. (2010). Large-diameter graphene nanotubes synthesized using Ni nanowire templates. Nano Lett. 10, 4844-4850. doi: 10.1021/nl102445x

Wang, T., Zhu, J., Chen, Y., Yang, H., Qin, Y., Li, F., et al. (2017). Large-scale production of silicon nanoparticles@graphene embedded in nanotubes as ultra-robust battery anodes. J. Mater. Chem. A. 5, 4809-4817. doi: 10.1039/C6TA10631E

Xu, T., Wang, D., Qiu, P., Zhang, J., Wang, Q., Xia, B., et al. (2018). In situ synthesis of porous $\mathrm{Si}$ dispersed in carbon nanotube intertwined expanded graphite for high-energy lithium-ion batterie. Nanoscale 10, 16638-16644. doi: $10.1039 / \mathrm{C} 8 \mathrm{NR} 04587 \mathrm{~A}$
Yan, Y., Xu, Z., Liu, C., Dou, H., Wei, J., Zhao, X., et al. (2019). Rational design of the robust janus shell on silicon anodes for high-performance lithium-ion batteries. ACS Appl. Mater. Interfaces 11, 17375-17383. doi: 10.1021/acsami.9b01909

Yue, X. Y., Yan, Z., Song, Y., Wu, X. J., and Zhou, Y. N. (2018). Synthesis of complementary hierarchical structured Si/C composites with high Si content for lithium-ion batteries. Nanoscale 10, 19195-19202. doi: 10.1039/C8NR04280B

Zhai, W., Ai, Q., Chen, L., Wei, S., Li, D., Zhang, L., et al. (2017). Walnut-inspired microsized porous silicon/graphene core-shell composites for high-performance lithium-ion battery anodes. Nano Res. 10, 4274-4283. doi: $10.1007 / \mathrm{s} 12274-017-1584-5$

Zhang, C., Kang, T.-H., Yu, J.-S. (2017). Three-dimensional spongy nanographenefunctionalized silicon anodes for lithium ion batteries with superior cycling stability. Nano Res. 11, 233-245. doi: 10.1007/s12274-017-1624-1

Zhang, H., Zong, P., Chen, M., Jin, H., Bai, Y., Li, S., et al. (2019). In situ synthesis of multilayer carbon matrix decorated with copper particles: enhancing the performance of $\mathrm{Si}$ as anode for Li-ion batteries. ACS Nano 13, 3054-3062. doi: 10.1021/acsnano.8b08088

Zhang, T., Wang, Y., Song, T., Miyaoka, H., Shinzato, K., Miyaoka, H., et al. (2018). Ammonia, a switch for controlling high ionic conductivity in lithium borohydride ammoniates. Cell Press 2, 1522-1533. doi: 10.1016/j.joule.2018.04.015

Zhou, M., Li, X., Wang, B., Zhang, Y., Ning, J., Xiao, Z., et al. (2015). High-performance silicon battery anodes enabled by engineering graphene assemblies. Nano Lett. 15, 6222-6228. doi: 10.1021/acs.nanolett.5b02697

Conflict of Interest: The authors declare that the research was conducted in the absence of any commercial or financial relationships that could be construed as a potential conflict of interest.

Copyright (c) 2020 Shi, Nie, Fan, Fu, Fang, Dou and Zhang. This is an open-access article distributed under the terms of the Creative Commons Attribution License (CC $B Y)$. The use, distribution or reproduction in other forums is permitted, provided the original author(s) and the copyright owner(s) are credited and that the original publication in this journal is cited, in accordance with accepted academic practice. No use, distribution or reproduction is permitted which does not comply with these terms. 